\title{
AN ESTIMATE FOR THE GAUSS-KRONECKER CURVATURE OF 3-DIMENSIONAL SMOOTH HYPERSURFACES IN $\mathbb{E}^{4}$
}

\author{
BOGDAN D. SUCEAVĂ
}

(Communicated by Kazım ILARSLAN)

\begin{abstract}
We give two alternate proofs of B.-Y. Chen's inequality with classical curvature invariants for 3-dimensional hypersurfaces in $\mathbb{E}^{4}$. Then, by using an idea described by Cvetkovski, we obtain an estimate of the Gauss-Kronecker curvature of a three-dimensional smooth hypersurface in the four dimensional Euclidean space in function of its mean curvature and its scalar curvature.
\end{abstract}

\section{INTRODUCTION}

There are many fundamental properties of real numbers that contribute essentially to the special geometric properties that the dimension 3 objects have. In the present paper we use the fact that the derivative of a cubic equation is a quadratic function, whose properties can be controlled. By using this fundamental fact, we prove an inequality in terms of curvature invariants of a hypersurface in a four dimensional Euclidean ambient.

First, we remind some classical notations in the differential geometry of smooth hypersurfaces. Let $\sigma: U \subset \mathbb{R}^{n} \rightarrow \mathbb{R}^{n+1}$ be a hypersurface given by the smooth map $\sigma$. Let $p$ be a point on the hypersurface. Denote $\sigma_{k}(p)=\frac{\partial \sigma}{\partial x_{k}}$, for all $k$ from 1 to $n$. Consider $\left\{\sigma_{1}(p), \sigma_{2}(p), \ldots, \sigma_{n}(p), N(p)\right\}$, the Gauss frame of the hypersurface, where $N$ denotes the normal vector field. We denote by $g_{i j}(p)$ the coefficients of the first fundamental form and by $h_{i j}(p)$ the coefficients of the second fundamental form. Then we have

$$
g_{i j}(p)=\left\langle\sigma_{i}(p), \sigma_{j}(p)\right\rangle, \quad h_{i j}(p)=\left\langle N(p), \sigma_{i j}(p)\right\rangle .
$$

The Weingarten map $L_{p}=-d N_{p} \circ d \sigma_{p}^{-1}: T_{\sigma(p)} \sigma \rightarrow T_{\sigma(p)} \sigma$ is linear. Denote by $\left(h_{j}^{i}(p)\right)_{1 \leq i, j \leq n}$ the matrix associated to Weingarten's map, that is:

$$
L_{p}\left(\sigma_{i}(p)=h_{i}^{k}(p) \sigma_{k}(p),\right.
$$

where the repeated index and upper script above indicates Einstein's summation convention. Weingerten's operator is self-adjoint, which implies that the roots of

Date: Received: April 3, 2014 and Accepted: May 27, 2014.

2010 Mathematics Subject Classification. Primary 53B25, 53B20; Secondary 53B21.

Key words and phrases. principal curvatures, scalar curvature, shape operator, umbilical hypersurfaces, mean curvature, Gauss-Kronecker curvature. 
the algebraic equation

$$
\operatorname{det}\left(h_{j}^{i}(p)-\lambda(p) \delta_{j}^{i}\right)=0
$$

are real. The eigenvalues of Weingarten's linear map are called principal curvatures of the hypersurface. They are the roots $\lambda_{1}(p), \lambda_{2}(p), \ldots, \lambda_{n}(p)$ of this algebraic equation. The mean curvature at the point $p$ is

$$
H(p)=\frac{1}{n}\left[\lambda_{1}(p)+\ldots+\lambda_{n}(p)\right]
$$

and the Gauss-Kronecker curvature is

$$
K(p)=\lambda_{1}(p) \lambda_{2}(p) \ldots \lambda_{n}(p) .
$$

If $M$ is a submanifold of a Riemannian manifold $\bar{M}$, and if their sectional curvatures are sec and $\overline{\mathrm{sec}}$, respectively, then from the Gauss equation (see e.g. [9], pg.131) we have:

$$
\sec \left(e_{i} \wedge e_{j}\right)-\overline{\sec }\left(e_{i} \wedge e_{j}\right)=\lambda_{i} \lambda_{j}
$$

If the ambient space is Euclidean, then $\overline{\sec }\left(e_{i} \wedge e_{j}\right)=0$ at every point, in every planar direction. This means that the scalar curvature of a hypersurface in Euclidean ambient space at point $p \in M^{n}$ is

$$
\operatorname{scal}(p)=\sum_{i<j} \sec \left(e_{i} \wedge e_{j}\right)=\sum_{i<j} \lambda_{i} \lambda_{j} .
$$

The normalized scalar curvature is $\rho=\left(\begin{array}{c}n \\ 2\end{array}\right)^{-1}$ scal.

In [13] it was proved that for a hypersurface $M^{n}$ in a Riemannian $(n+1)$-manifold $\bar{M}^{n+1}$, at every point $p \in M$ the following inequality holds:

$$
\operatorname{scal}(p) \leq \frac{n(n-1)}{2} H^{2}+\sum_{i<j} \overline{\sec }\left(e_{i} \wedge e_{j}\right),
$$

where scal is the scalar curvature of $M$ at $p, H$ is the mean curvature at $p$, and $\overline{\sec }\left(e_{i} \wedge e_{j}\right)$ is the sectional curvature on the plane generated by vectors $e_{i}$ and $e_{j}$ tangent to the ambient space $\bar{M}$.

The equality holds at $p$ if and only if $p$ is an umbilical point.

This inequality was also studied in [12]. For a general formulation in pseudoRiemannian ambient spaces, see e.g. [6] pp.36-37, where the same fact is derived as a consequence of Gauss' equation and Cauchy-Schwarz inequality. In [13] it is shown how from the inequality (1.1) one may derive Chen's inequality with classical curvature invariants, proved originally by B.-Y. Chen in 1996.

Theorem 1.1. [3] Let $\rho=2 \operatorname{scal}(p) / n(n-1)$ denote the normalized scalar curvature of a hypersurface $M^{n}$ isometrically immersed in a Riemannian space form $R^{n+1}(\epsilon)$. Then we have the inequality:

$$
\rho \leq H^{2}+\epsilon
$$

at every point $p \in M$.

The equality holds if and only if $p$ is an umbilical point.

The general form of this result, for submanifolds of arbitrary codimension, is Proposition 2.5 in [6].

In the particular case when the ambient space is Euclidean, we have $\epsilon=0$. We provide below two alternate proof for this inequality for three-dimensional hypersurfaces in four-dimensional Euclidean ambient space. For further discussion see also [15]. 
This present development is a natural continuation of the study of new curvature invariants, as it was inspired by the ideas considered by B.-Y. Chen in $[2,4,5]$. For the whole vision of this research direction, the most comprehensive reference is the recent monograph [6]. For recent explorations of various inequalities of algebraic or analytic inspiration that yield geometric consequences in terms of curvature invariants, see $[1,7,14]$, all inspired by the philosophy developed in [6].

\section{Chen's Fundamental Inequality with Classical Curvature INVARIANTS FOR HyPERSURFACES OF DimENSION THREE}

We focus now on a particular case of Theorem 1.1, namely the following.

Corollary 2.1. Let $M^{3} \subset \mathbb{R}^{4}$ be a smooth hypersurface and $\lambda_{1}, \lambda_{2}, \lambda_{3}$ be its principal curvatures in the ambient space $\mathbb{R}^{4}$ endowed with the canonical metric. Let $p \in M$ be an arbitrary point. Denote by $H(p)=\frac{1}{3}\left(\lambda_{1}+\lambda_{2}+\lambda_{3}\right)$ the mean curvature, by $\operatorname{scal}(p)=\sec \left(e_{1} \wedge e_{2}\right)+\sec \left(e_{3} \wedge e_{1}\right)+\sec \left(e_{2} \wedge e_{3}\right)=$ $\lambda_{1}(p) \lambda_{2}(p)+\lambda_{3}(p) \lambda_{1}(p)+\lambda_{2}(p) \lambda_{3}(p)$ the scalar curvature at the point $p \in M^{3}$. Then

$$
H^{2}(p) \geq \rho(p),
$$

with equality if and only if the point is umbilical, i.e. when $\lambda_{1}(p)=\lambda_{2}(p)=\lambda_{3}(p)$.

Alternate proof 1: We have

$$
\begin{gathered}
9 H^{2}=(3 H)^{2}=\left(\lambda_{1}+\lambda_{2}+\lambda_{3}\right)^{2}=\lambda_{1}^{2}+\lambda_{2}^{2}+\lambda_{3}^{2}+2\left(\lambda_{1} \lambda_{2}+\lambda_{3} \lambda_{1}+\lambda_{2} \lambda_{3}\right) \geq \\
\geq 3\left(\lambda_{1} \lambda_{2}+\lambda_{3} \lambda_{1}+\lambda_{2} \lambda_{3}\right),
\end{gathered}
$$

this last inequality holds true since

$$
\lambda_{1}^{2}+\lambda_{2}^{2}+\lambda_{3}^{2} \geq \lambda_{1} \lambda_{2}+\lambda_{3} \lambda_{1}+\lambda_{2} \lambda_{3}
$$

and in this inequality equality holds if and only if $\lambda_{1}=\lambda_{2}=\lambda_{3}$. From Gauss's equation, we express the scalar curvature of the hypersurface $M^{3}$ as

$$
\text { scal }=\lambda_{1} \lambda_{2}+\lambda_{3} \lambda_{1}+\lambda_{2} \lambda_{3}
$$

This yields $9 H^{2} \geq 3$ scal. The normalized scalar curvature in dimension three is $\rho=\frac{1}{3} s c a l$, therefore $H^{2} \geq \rho$, with equality if and only if the point is an umbilic.

Alternate proof 2: For some arbitrary point $p \in M^{3}$, consider the cubic equation that has as roots the real numbers $\lambda_{1}, \lambda_{2}, \lambda_{3}$. Namely, by using Viète's formulae, consider:

$$
f(x)=x^{3}-3 H x^{2}+s c a l \cdot x-K=0 .
$$

This is a differentiable function in the variable $x$, and its derivative is

$$
f^{\prime}(x)=3 x^{2}-6 H x+s c a l=0 .
$$

Since the variable $x$ is real, the discriminant of this quadratic function must be nonnegative, thus

$$
36 H^{2}-4 \cdot 3 \cdot s c a l \geq 0,
$$

thus $3 H^{2}-$ scal $\geq 0$, which means we obtain again $H^{2} \geq \rho$. 


\section{An Estimate for Gauss-Kronecker Curvature}

In this section we apply the technique described by Cvetkovski in Chapter 15 in his recent interesting volume [8] to prove the following.

Theorem 3.1. Let $M^{3} \subset \mathbb{R}^{4}$ be a smooth hypersurface and $\lambda_{1}, \lambda_{2}, \lambda_{3}$ be its principal curvatures in the ambient space $\mathbb{R}^{4}$ endowed with the canonical metric. Let $p \in M$ be an arbitrary point. Denote by $H(p)=\frac{1}{3}\left(\lambda_{1}+\lambda_{2}+\lambda_{3}\right)$ the mean curvature, by $\operatorname{scal}(p)=\sec \left(e_{1} \wedge e_{2}\right)+\sec \left(e_{3} \wedge e_{1}\right)+\sec \left(e_{2} \wedge e_{3}\right)=\lambda_{1}(p) \lambda_{2}(p)+\lambda_{3}(p) \lambda_{1}(p)+$ $\lambda_{2}(p) \lambda_{3}(p)$ the scalar curvature at the point $p \in M^{3}$, and by $K(p)=\lambda_{1}(p) \lambda_{2}(p) \lambda_{3}(p)$ the Gauss-Kronecker curvature of the hypersurface. Then

$$
\left(H+\frac{q}{3}\right)\left(-H^{2}-\frac{1}{3} H q+\frac{2}{3} s c a l\right) \leq K \leq\left(H-\frac{q}{3}\right)\left(-H^{2}+\frac{1}{3} H q+\frac{2}{3} \text { scal }\right),
$$

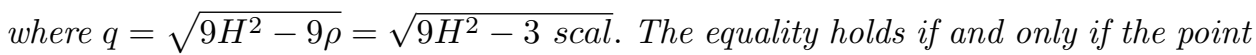
is semi-umbilical, i.e. if the following holds

$$
\left(\lambda_{1}(p)-\lambda_{2}(p)\right)\left(\lambda_{3}(p)-\lambda_{1}(p)\right)\left(\lambda_{2}(p)-\lambda_{3}(p)\right)=0 .
$$

Proof: Note first that $9 H^{2}-9 \rho \geq 0$ is a consequence of Chen's fundamental inequality with classical curvature invariants, explored in the previous section, so it makes sense to take $q^{2}=9 H^{2}-9 \rho$.

If $q=0$ at $p \in M$, then, by the equality case in Chen's fundamental inequality with classical curvature invariants, $p$ is an umbilical point. In this case, the double inequality in the statement turns out to be an identity:

$$
K=-H^{3}+\frac{2}{3} \mathrm{scal} \cdot H,
$$

which reduces immediately to $2 \lambda^{3}=2 \lambda^{3}$.

Now assume that $p$ is not an umbilical point, i.e. $q>0$ at $p \in M$.

Consider the function given by the real polynomial of degree three whose roots at every point at the principal curvatures of the hypersurface:

$$
f(x)=\left(x-\lambda_{1}\right)\left(x-\lambda_{2}\right)\left(x-\lambda_{3}\right)=x^{3}-3 H x^{2}+s c a l \cdot x-K .
$$

Then the derivative of $f$ is the quadratic function

$$
f^{\prime}(x)=3 x^{2}-6 H x+s c a l .
$$

The equation $f^{\prime}(x)=0$ has the real roots:

$$
x_{1,2}=\frac{6 H \pm \sqrt{36 H^{2}-12 \cdot s c a l}}{6}=H \pm \frac{q}{3} .
$$

Denote by $x_{1}=H+\frac{q}{3}$, and by $x_{2}=H-\frac{q}{3}$, that is $x_{2} \leq x_{1}$. Remark that the second derivative is

$$
f^{\prime \prime}(x)=6 x-6 H,
$$

and this yields

$$
f^{\prime \prime}\left(x_{1}\right)=6\left(H+\frac{q}{3}\right)-6 H=2 q>0,
$$

which means that $f$ has a local minimum at $x_{1}$. Similarly,

$$
f^{\prime \prime}\left(x_{2}\right)=6\left(H-\frac{q}{3}\right)-6 H=-2 q<0,
$$

and this means that the original function $f$ has a local maximum at $x_{2}$. So, $f$ is a polynomial of degree three, with three real roots. This means the local minimum 
must have a nonpositive value, while the local maximum must have a nonnegative value:

$$
f\left(\frac{3 H-q}{3}\right) \geq 0, \quad f\left(\frac{3 H+q}{3}\right) \leq 0 .
$$

By evaluating $f$ at these two values corresponding to $x_{2}$ and $x_{1}$, respectively, we have:

$$
\begin{aligned}
& \left(\frac{3 H-q}{3}\right)^{3}-3 H\left(\frac{3 H-q}{3}\right)^{2}+\text { scal } \cdot\left(\frac{3 H-q}{3}\right)-K \geq 0, \\
& \left(\frac{3 H+q}{3}\right)^{3}-3 H\left(\frac{3 H+q}{3}\right)^{2}+\text { scal } \cdot\left(\frac{3 H+q}{3}\right)-K \leq 0 .
\end{aligned}
$$

By joining these two inequalities, we have:

$$
\begin{gathered}
\left(\frac{3 H+q}{3}\right)^{3}-3 H\left(\frac{3 H+q}{3}\right)^{2}+\text { scal } \cdot\left(\frac{3 H+q}{3}\right) \leq K \leq \\
\leq\left(\frac{3 H-q}{3}\right)^{3}-3 H\left(\frac{3 H-q}{3}\right)^{2}+\text { scal } \cdot\left(\frac{3 H-q}{3}\right) .
\end{gathered}
$$

A direct computation shows that the upper bound is:

$$
\begin{aligned}
& \left(H-\frac{q}{3}\right)\left[\left(H-\frac{q}{3}\right)^{2}-3 H\left(H-\frac{q}{3}\right)+\text { scal }\right]= \\
= & \left(H-\frac{q}{3}\right)\left[H^{2}-\frac{2}{3} H q+\frac{q^{2}}{9}-3 H^{2}+H q+\text { scal }\right]= \\
= & \left(H-\frac{q}{3}\right)\left[-2 H^{2}+\frac{1}{3} H q+\frac{9 H^{2}-3 \cdot \text { scal }}{9}+\text { scal }\right] .
\end{aligned}
$$

This last expression reduces to the right hand side term in the double inequality stated in the Theorem. The expression in the lower bound is obtained by a similar computation.

To complete the proof, we need to discuss now when the equality holds in the two stated inequalities. Equality holds in either of the two inequalities when we have either

$$
f\left(\frac{3 H-q}{3}\right)=0,
$$

or

$$
f\left(\frac{3 H+q}{3}\right)=0 \text {. }
$$

Suppose $f\left(\frac{3 H-q}{3}\right)=0$. Then $f(x)=\left(x-\lambda_{1}\right)\left(x-\lambda_{2}\right)\left(x-\lambda_{3}\right)$, which means that $\frac{3 H-q}{3}$ should be one of the roots. Suppose $\frac{3 H-q}{3}=\lambda_{1}$. Then we get $\lambda_{2}+\lambda_{3}-q=2 \lambda_{1}$. By squaring both sides in

$$
\lambda_{2}+\lambda_{3}-2 \lambda_{1}=\sqrt{9 H^{2}-3 \cdot s c a l}
$$

we obtain

$$
3 \lambda_{1}^{2}+3 \lambda_{2} \lambda_{3}-3 \lambda_{1} \lambda_{2}-3 \lambda_{1} \lambda_{3}=0,
$$

which factors as $\left(\lambda_{1}-\lambda_{2}\right)\left(\lambda_{1}-\lambda_{3}\right)=0$, which means that at least a second principal curvature $\lambda_{2}$ or $\lambda_{3}$ must equal $\lambda_{1}$, and this can be written as

$$
\left(\lambda_{1}(p)-\lambda_{2}(p)\right)\left(\lambda_{3}(p)-\lambda_{1}(p)\right)\left(\lambda_{2}(p)-\lambda_{3}(p)\right)=0 .
$$

The case $f\left(\frac{3 H+q}{3}\right)=0$ yields a similar conclusion. 
There are many examples of hypersurfaces satisfying the semi-umbilicity condition at every point, e.g. $S^{2} \times \mathbb{R}$, or $S^{1} \times \mathbb{R}^{2}$.

A nontrival example is mentioned by Hasanis and Vlachos in [11], in Proposition 4.1. In their example, for that class of hypersurfaces in $\mathbb{R}^{4}$, the principal curvatures are $\lambda_{1}=\lambda_{2}=\frac{9 H}{4}$, and $\lambda_{3}=-\frac{3 H}{2}$.

It is possible that some techniques in the classical theory of inequalities, as it is presented in classical references e.g. [10], may yield inequalities whose geometric interpretation encodes information in terms of curvature invariants. Some of these connections are still to be explored.

\section{REFERENCES}

[1] Brzycki, B., Giesler, M., Gomez, K., Odom, L. H. and Suceavă, B. D., A Ladder of curvatures for hypersurfaces in Euclidean ambient space, to appear in Houston $\mathrm{J}$. Math.

[2] Chen, B.-Y., Some pinching and classification theorems for minimal submanifolds, Arch. Math., 60 (1993), 568-578.

[3] Chen, B.-Y., Mean curvature and shape operator of isometric immersions in real-spaceforms, Glasgow Math.J. 38 (1996), 87-97.

[4] Chen, B.-Y., Relations between Ricci curvature and shape operator for submanifolds with arbitrary codimension, Glasgow Math. J., 41 (1999), 33-41.

[5] Chen, B.-Y., Some new obstructions to minimal and Lagrangian isometric immersions, Japanese J. Math., 26 (2000), 105-127.

[6] Chen, B.-Y., Pseudo-Riemannian geometry, $\delta$-invariants and applications, World Scientific, 2011.

[7] Conley, C. T. R., Etnyre, R., Gardener, B., Odom, L. H. and Suceavă, B. D., New Curvature Inequalities for Hypersurfaces in the Euclidean Ambient Space, Taiwanese J. Math., 17 (3) (2013), 885-895.

[8] Cvetkovski, Z., Inequalities. Theorems, Techniques and Selected Problems, SpringerVerlag, 2012.

[9] do Carmo, M. P., Riemannian Geometry, Birkhäuser, 1992.

[10] Hardy, G. H., Littlewood, J. E. and Pólya, G., Inequalities (Cambridge Mathematical Library), Cambridge University Press; 2 edition, 1988.

[11] Hasanis, Th. and Vlachos, Th., Hypersurfaces in $\mathbb{E}^{4}$ with harmonic mean curvature vector field, Math. Nachr. 172 (1995), 145-169.

[12] Hong, S., Matsumoto, K. and Tripathi, M., Certain basic inequalities for submanifolds of locally conformal Kaehler space forms, Sci. Univ. Tokyo Journal of Mathematics, Vol. 41, No. 1 (2005), 75-94.

[13] Suceavă, B. D., Some remarks on B.-Y. Chen's inequality involving classical invariants, Anal. Sti. Univ. "Al.I.Cuza" Iasi, s.I.a, Math., 64 (1999), 405-412.

[14] Suceavă, B. D., The amalgamatic curvature and the orthocurvatures of three dimensional hypersurfaces in $\mathbb{E}^{4}$ (to appear).

[15] Suceavă, B. D. and Vajiac, M. B., Remarks on Chen's fundamental inequality with classical curvature invariants in Riemannian Spaces, Annals Sti. Univ. "Al. I. Cuza", s.I.a, Math. 54 (2008), no. 1, pp. 27-37.

Department of Mathematics, California State University, Fullerton, CA 91834-6850, U.S.A.

E-mail address: bsuceava@fullerton.edu 